Annals of Pure and Applied Mathematics

Vol. 13, No. 2, 2017, 151-163

ISSN: 2279-087X (P), 2279-0888(online)

Published on 1 April 2017

www.researchmathsci.org

DOI: http://dx.doi.org/10.22457/apam.v13n2al

Annals of

Pure and Applied Mathematics

\title{
Edge Trimagic Total Labeling of Mobius Ladder, Book and Dragon Graphs
}

\author{
C. Jayasekaran ${ }^{1}$ and J. Little Flower ${ }^{2}$ \\ ${ }^{1}$ Department of Mathematics, Pioneer Kumaraswamy College \\ Nagercoil - 629 003, Tamilnadu, India. \\ Corresponding author. E-mail: jaya_pkc@yahoo.com \\ ${ }^{2}$ Department of Mathematics, Arignar Anna College, Aralvaimozhi - 629301 \\ Tamilnadu, India. Email: littleflowerj.levin@yahoo.com
}

Received 20 February 2017; accepted 14 March 2017

Abstract. A graph $\mathrm{G}=(\mathrm{V}, \mathrm{E})$ with $\mathrm{p}$ vertices and $\mathrm{q}$ edges is said to be edge trimagic total labeling if there exists a bijection $\mathrm{f}: \mathrm{V}(\mathrm{G}) \cup \mathrm{E}(\mathrm{G}) \rightarrow\{1,2, \ldots, \mathrm{p}+\mathrm{q}\}$ such that for each edge $u v \in E(G)$, the value of $f(u)+f(u v)+f(v)$ is either $k_{1}$ or $k_{2}$ or $k_{3}$. In this paper, we prove that the graphs $(\mathrm{n}, l)$ - dragon, Mobius ladder $\mathrm{M}_{\mathrm{n}}$ and Book $\mathrm{B}_{\mathrm{n}}$ are edge trimagic total labeling.

Keywords: Graph, function, bijection, dragon, mobius ladder, book, trimagic labeling

AMS Mathematics Subject Classification (2010): 05 C78

\section{Introduction}

A labeling of a graph is an assignment of integers to vertices or sometimes edges in a graph based upon certain criteria. Rosa in 1967, introduced the concept of graph labeling. Any graph labeling will have the following three common characteristics: (i) A set of numbers from which vertex labels are chosen (ii) A rule that assigns a value to each edge (iii) A condition that this value has to satisfy [13]. Graph labeling are of many types such as graceful, harmonious, elegant, cordial, magic, antimagic, bimagic etc. Harary [4] is referred to know about the notations in graph theory.

Magic labeling was introduced by Sedlacek [12]. Kotzing and Rosa [10], defined edge magic of a graph $\mathrm{G}$ with a bijection $\mathrm{f}: \mathrm{V} \cup \mathrm{E} \rightarrow\{1,2, \ldots, \mathrm{p}+\mathrm{q}\}$ such that, for each edge $u v \in E(G), f(u)+f(u v)+f(v)$ is a magic constant. In [3] shows the cycle $C_{n}$ with $P_{3}$ chords are edge magic total labeling. Edge bimagic labeling of graphs was introduced by Babujee [2] in 2004, defined by a graph $G$ with a bijection $f: V \cup E \rightarrow\{1,2, \ldots, p+q\}$ such that for each edge $u v \in E(G)$, the value of $f(u)+f(u v)+f(v)$ is either $k_{1}$ or $k_{2}$. Magic and bimagic labeling for disconnected graphs are showed in [1].

In 2013, Jayasekaran et al. [5] introduced the edge Trimagic total labeling of graphs. An edge trimagic total labeling of a (p, q) graph $G$ is a bijection $f: V \cup E \rightarrow\{1,2$, $\ldots, p+q\}$ such that for each edge $u v \in E(G)$, the value of $f(u)+f(u v)+f(v)$ is equal to any of the distinct constants $\mathrm{k}_{1}$ or $\mathrm{k}_{2}$ or $\mathrm{k}_{3}$. An edge trimagic total labeling is called a super edge trimagic total labeling of a graph $\mathrm{G}$, if the vertices are labeled with the 


\section{Jayasekaran and J. Little Flower}

smallest possible integers i.e. $1,2, \ldots$, p. Graphs such as Umbrella $\mathrm{U}_{\mathrm{n}, \mathrm{m}}$, Dumb bell $\mathrm{Db}_{\mathrm{n}}$ and Circular ladder CL(n) are proved to be edge trimagic and super edge trimagic total graphs in [7]. In [6], edge trimagic labeling of digraphs were discussed.

A $(\mathrm{n}, l)$ - dragon is formed by joining an end point of a path $\mathrm{P}_{\mathrm{n}}$ to a point of cycle $\mathrm{C}_{\mathrm{n}}$ (Koh, et al [11] call these tadpoles; Kim and Park [9] call them kites). Mobius ladder $M_{n}$ is the graph obtained from the ladder $P_{n} \times P_{2}$ by joining the opposite end points of the two copies of $P_{n}$. The Book $B_{n}$ is the graph $S_{n} \times P_{2}$ where $S_{n}$ is the star with $n+1$ vertices.

For more references, we use dynamic survey of graph labeling by Gallian [8]. In this paper, we prove that the dragon, Mobius ladder and Book are edge trimagic total labeling graphs.

\section{Main results}

Theorem 2.1. The Mobius ladder $M_{n}$ is an edge trimagic for odd $n$.

Proof: Let $V=\left\{u_{i}, v_{i} / 1 \leq i \leq n\right\}$ be the vertex set and $E=\left\{u_{i} u_{i+1}, v_{i} v_{i+1}\right.$ $/ 1 \leq \mathrm{i} \leq \mathrm{n}-1\} \cup\left\{\mathrm{u}_{\mathrm{i}} \mathrm{v}_{\mathrm{i}} / 1 \leq \mathrm{i} \leq \mathrm{n}\right\} \cup\left\{\mathrm{u}_{1} \mathrm{v}_{\mathrm{n}}, \mathrm{v}_{\mathrm{l}} \mathrm{u}_{\mathrm{n}}\right\}$ be the edge set of the Mobius ladder $\mathrm{M}_{\mathrm{n}}$. Then $M_{n}$ has $2 n$ vertices and $3 n$ edges. Define a bijection $f: V \cup E \rightarrow\{1,2, \ldots, 5 n\}$ such that

$$
\begin{aligned}
& \mathrm{f}\left(\mathrm{u}_{\mathrm{i}}\right)=\left\{\begin{array}{l}
\frac{\mathrm{i}+1}{2}, 1 \leq \mathrm{i} \leq \mathrm{n} \text { and } \mathrm{i} \text { is odd } \\
\frac{\mathrm{n}+\mathrm{i}+1}{2}, 1 \leq \mathrm{i} \leq \mathrm{n} \text { and } \mathrm{i} \text { is even }
\end{array}\right. \\
& \mathrm{f}\left(\mathrm{v}_{\mathrm{i}}\right)=\left\{\begin{array}{l}
\mathrm{n}+\frac{\mathrm{n}+\mathrm{i}}{2}, 1 \leq \mathrm{i} \leq \mathrm{n} \text { and } \mathrm{i} \text { is odd } \\
\mathrm{n}+\frac{\mathrm{i}}{2}, 1 \leq \mathrm{i} \leq \mathrm{n} \text { and } \mathrm{i} \text { is even }
\end{array}\right.
\end{aligned}
$$

and $\mathrm{f}\left(\mathrm{u}_{\mathrm{i}} \mathrm{u}_{\mathrm{i}+1}\right)=5 \mathrm{n}-\mathrm{i}-1,1 \leq \mathrm{i} \leq \mathrm{n}-1 ; \mathrm{f}\left(\mathrm{v}_{\mathrm{i}} \mathrm{v}_{\mathrm{i}+1}\right)=3 \mathrm{n}-\mathrm{i}, 1 \leq \mathrm{i} \leq \mathrm{n}-1 ; \mathrm{f}\left(\mathrm{u}_{\mathrm{i}} \mathrm{v}_{\mathrm{i}}\right)=4 \mathrm{n}-\mathrm{i}, 1 \leq \mathrm{i} \leq \mathrm{n} ; \mathrm{f}\left(\mathrm{u}_{1} \mathrm{v}_{\mathrm{n}}\right)$ $=5 \mathrm{n}-1$ and $\mathrm{f}\left(\mathrm{v}_{1} \mathrm{u}_{\mathrm{n}}\right)=5 \mathrm{n}$.

To prove this labeling is an edge trimagic total labeling.

For the edge $u_{1} v_{n}, f\left(u_{1}\right)+f\left(u_{1} v_{n}\right)+f\left(v_{n}\right)=1+5 n-1+2 n=7 n=\lambda_{1}$.

For the edge $v_{1} u_{n}, f\left(v_{1}\right)+f\left(v_{1} u_{n}\right)+f\left(u_{n}\right)=n+\frac{n+1}{2}+5 n+\frac{n+1}{2}=7 n+1=\lambda_{2}$.

Consider the edges $\mathrm{u}_{\mathrm{i}} \mathrm{u}_{\mathrm{i}+1}, 1 \leq \mathrm{i} \leq \mathrm{n}-1$.

For odd $\mathrm{i}, \mathrm{f}\left(\mathrm{u}_{\mathrm{i}}\right)+\mathrm{f}\left(\mathrm{u}_{\mathrm{i}} \mathrm{u}_{\mathrm{i}+1}\right)+\mathrm{f}\left(\mathrm{u}_{\mathrm{i}+1}\right)=\frac{\mathrm{i}+1}{2}+5 \mathrm{n}-\mathrm{i}-1+\frac{\mathrm{n}+\mathrm{i}}{2}+1=\frac{11 \mathrm{n}+1}{2}=\lambda_{3}$.

For even $i, f\left(u_{i}\right)+f\left(u_{i} u_{i+1}\right)+f\left(u_{i+1}\right)=\frac{n+i+1}{2}+5 n-i-1+\frac{i}{2}+1=\frac{11 n+1}{2}=\lambda_{3}$.

Consider the edges $\mathrm{v}_{\mathrm{i}} \mathrm{v}_{\mathrm{i}+1}, 1 \leq \mathrm{i} \leq \mathrm{n}-1$.

For odd $\mathrm{i}, \mathrm{f}\left(\mathrm{v}_{\mathrm{i}}\right)+\mathrm{f}\left(\mathrm{v}_{\mathrm{i}} \mathrm{v}_{\mathrm{i}+1}\right)+\mathrm{f}\left(\mathrm{v}_{\mathrm{i}+1}\right)=\mathrm{n}+\frac{\mathrm{n}+\mathrm{i}}{2}+3 \mathrm{n}-\mathrm{i}+\mathrm{n}+\frac{\mathrm{i}+1}{2}=\frac{11 \mathrm{n}+1}{2}=\lambda_{3}$.

For even $\mathrm{i}, \mathrm{f}\left(\mathrm{v}_{\mathrm{i}}\right)+\mathrm{f}\left(\mathrm{v}_{\mathrm{i}} \mathrm{v}_{\mathrm{i}+1}\right)+\mathrm{f}\left(\mathrm{v}_{\mathrm{i}+1}\right)=\mathrm{n}+\frac{\mathrm{i}}{2}+3 \mathrm{n}-\mathrm{i}+\mathrm{n}+\frac{\mathrm{n}+\mathrm{i}+1}{2}=\frac{11 \mathrm{n}+1}{2}=\lambda_{3}$. 
Edge Trimagic Total Labeling of Mobius Ladder, Book and Dragon Graphs

Consider the edges $\mathrm{u}_{\mathrm{i}} \mathrm{v}_{\mathrm{i}}, 1 \leq \mathrm{i} \leq \mathrm{n}$.

For odd $\mathrm{i}, \mathrm{f}\left(\mathrm{u}_{\mathrm{i}}\right)+\mathrm{f}\left(\mathrm{u}_{\mathrm{i}} \mathrm{v}_{\mathrm{i}}\right)+\mathrm{f}\left(\mathrm{v}_{\mathrm{i}}\right)=\frac{\mathrm{i}+1}{2}+4 \mathrm{n}-\mathrm{i}+\mathrm{n}+\frac{\mathrm{n}+\mathrm{i}}{2}=\frac{11 \mathrm{n}+1}{2}=\lambda_{3}$.

For even $\mathrm{i}, \mathrm{f}\left(\mathrm{u}_{\mathrm{i}}\right)+\mathrm{f}\left(\mathrm{u}_{\mathrm{i}} \mathrm{v}_{\mathrm{i}}\right)+\mathrm{f}\left(\mathrm{v}_{\mathrm{i}}\right)=\frac{\mathrm{n}+\mathrm{i}+1}{2}+4 \mathrm{n}-\mathrm{i}+\mathrm{n}+\frac{\mathrm{i}}{2}=\frac{11 \mathrm{n}+1}{2}=\lambda_{3}$.

Hence for each edge $u v \in E, f(u)+f(u v)+f(v)$ yields any one of the magic constants $\lambda_{1}=$ $7 \mathrm{n}, \lambda_{2}=7 \mathrm{n}+1$ and $\lambda_{3}=\frac{11 \mathrm{n}+1}{2}$. Therefore, the Mobius ladder $\mathrm{M}_{\mathrm{n}}$ is an edge trimagic total labeling for odd $n$.

Corollary 2.2. The Mobius ladder $M_{n}$ is super edge trimagic total labeling for odd $n$.

Proof: We proved that the Mobius ladder $M_{n}$ is an edge trimagic total labeling for odd $n$ with $2 \mathrm{n}$ vertices. The labeling given in Theorem 2.1 is as follows:

$$
\begin{gathered}
f\left(u_{i}\right)=\left\{\begin{array}{l}
\frac{\mathrm{i}+1}{2}, 1 \leq \mathrm{i} \leq \mathrm{n} \text { and } \mathrm{i} \text { is odd } \\
\frac{\mathrm{n}+\mathrm{i}+1}{2}, 1 \leq \mathrm{i} \leq \mathrm{n} \text { and } \mathrm{i} \text { is even }
\end{array}\right. \\
\mathrm{f}\left(\mathrm{v}_{\mathrm{i}}\right)=\left\{\begin{array}{l}
\mathrm{n}+\frac{\mathrm{n}+\mathrm{i}}{2}, 1 \leq \mathrm{i} \leq \mathrm{n} \text { and } \mathrm{i} \text { is odd } \\
\mathrm{n}+\frac{\mathrm{i}}{2}, 1 \leq \mathrm{i} \leq \mathrm{n} \text { and } \mathrm{i} \text { is even }
\end{array}\right.
\end{gathered}
$$

Hence the $2 \mathrm{n}$ vertices get labels $1,2, \ldots, 2 \mathrm{n}$. Therefore, the Mobius ladder $\mathrm{M}_{\mathrm{n}}$ is super edge trimagic total labeling for odd $n$.

Example 2.3. An edge trimagic total labeling of the Mobius ladder $\mathrm{M}_{7}$ is given in figure 1.

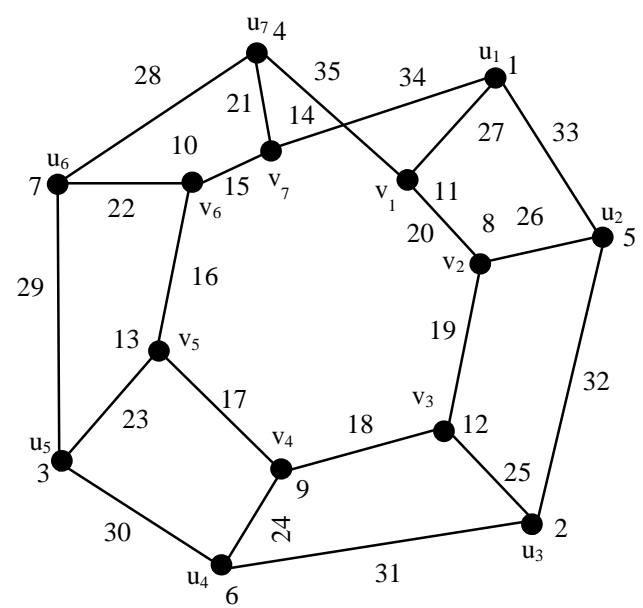

Figure1: $\mathrm{M}_{7}$ with $\lambda_{1}=49, \lambda_{2}=50$ and $\lambda_{3}=39$ 


\section{Jayasekaran and J. Little Flower}

Theorem 2.4. The Book $B_{n}$ is an edge trimagic graph.

Proof: Let $\mathrm{V}=\left\{\mathrm{v}_{\mathrm{o}}, \mathrm{u}_{\mathrm{o}}, \mathrm{v}_{\mathrm{i}}, \mathrm{u}_{\mathrm{i}} / 1 \leq \mathrm{i} \leq \mathrm{n}\right\}$ be the vertex set and $\mathrm{E}=\left\{\mathrm{v}_{\mathrm{o}} \mathrm{v}_{\mathrm{i},}, \mathrm{u}_{\mathrm{o}} \mathrm{u}_{\mathrm{i}} / 1 \leq \mathrm{i} \leq \mathrm{n}\right\} \cup\left\{\mathrm{u}_{\mathrm{o}} \mathrm{v}_{\mathrm{o}}, \mathrm{u}_{\mathrm{i}} \mathrm{v}_{\mathrm{i}}\right.$ $/ 1 \leq i \leq n\}$ be the edge set of the graph $B_{n}$. Then $B_{n}$ has $2 n+2$ vertices and $3 n+1$ edges.

Case 1. $\mathrm{n}$ is even.

Define a bijection $\mathrm{f}: \mathrm{V} \cup \mathrm{E} \rightarrow\{1,2,3, \ldots, 5 \mathrm{n}+3\}$ such that $\mathrm{f}\left(\mathrm{v}_{\mathrm{o}}\right)=1 ; \mathrm{f}\left(\mathrm{u}_{\mathrm{o}}\right)=\mathrm{n}+2 ; \mathrm{f}\left(\mathrm{v}_{\mathrm{i}}\right)=2 \mathrm{n}+3-\mathrm{i}, 1 \leq \mathrm{i} \leq \mathrm{n} ;$

$\mathrm{f}\left(\mathrm{u}_{\mathrm{i}}\right)=\left\{\begin{array}{l}2 \mathrm{i}, \quad 1 \leq \mathrm{i} \leq \frac{\mathrm{n}}{2} \\ 2 \mathrm{i}-\mathrm{n}+1, \quad \frac{\mathrm{n}}{2}+1 \leq \mathrm{i} \leq \mathrm{n}\end{array}\right.$

$\mathrm{f}\left(\mathrm{v}_{\mathrm{o}} \mathrm{v}_{\mathrm{i}}\right)=4 \mathrm{n}+\mathrm{i}+2,1 \leq \mathrm{i} \leq \mathrm{n} ; \mathrm{f}\left(\mathrm{u}_{1} \mathrm{v}_{1}\right)=3 \mathrm{n}+2$ and $\mathrm{f}\left(\mathrm{u}_{\mathrm{o}} \mathrm{v}_{\mathrm{o}}\right)=5 \mathrm{n}+3$.

$f\left(u_{0} u_{i}\right)= \begin{cases}4 n-2 i+4, & 1 \leq i \leq \frac{n}{2} \\ 5 n-2 i+3, & \frac{n}{2}+1 \leq i \leq n\end{cases}$

$f\left(u_{i} v_{i}\right)= \begin{cases}\frac{5}{2} n+3-i, & 2 \leq i \leq \frac{n}{2} \\ \frac{7}{2} n+2-i, & \frac{n}{2}+1 \leq i \leq n\end{cases}$

To prove this labeling is an edge trimagic total labeling.

For the edges $v_{0} v_{i}, 1 \leq i \leq n, f\left(v_{0}\right)+f\left(v_{0} v_{i}\right)+f\left(v_{i}\right)=1+4 n+i+2+2 n+3-i=6(n+1)=\lambda_{1}$.

For the edge $v_{0} u_{0}, f\left(v_{o}\right)+f\left(v_{0} u_{o}\right)+f\left(u_{o}\right)=1+5 n+3+n+2=6(n+1)=\lambda_{1}$.

Now consider the edges $\mathrm{u}_{\mathrm{o}} \mathrm{u}_{\mathrm{i}}, 1 \leq \mathrm{i} \leq \mathrm{n}$.

For $1 \leq \mathrm{i} \leq \frac{\mathrm{n}}{2}, \mathrm{f}\left(\mathrm{u}_{\mathrm{o}}\right)+\mathrm{f}\left(\mathrm{u}_{\mathrm{o}} \mathrm{u}_{\mathrm{i}}\right)+\mathrm{f}\left(\mathrm{u}_{\mathrm{i}}\right)=\mathrm{n}+2+4 \mathrm{n}-2 \mathrm{i}+4+2 \mathrm{i}=5 \mathrm{n}+6=\lambda_{2}$.

For $\frac{n}{2}+1 \leq i \leq n, f\left(u_{o}\right)+f\left(u_{o} u_{i}\right)+f\left(u_{i}\right)=n+2+5 n-2 i+3+2 i-n+1=5 n+6=\lambda_{2}$.

For the edge $v_{1} u_{1}, f\left(v_{1}\right)+f\left(v_{1} u_{1}\right)+f\left(u_{1}\right)=2 n+2+3 n+2+2=5 n+6=\lambda_{2}$.

Consider the edges $u_{i} v_{i}, 2 \leq \mathrm{i} \leq n$.

For $2 \leq \mathrm{i} \leq \frac{\mathrm{n}}{2}, \mathrm{f}\left(\mathrm{u}_{\mathrm{i}}\right)+\mathrm{f}\left(\mathrm{u}_{\mathrm{i}} \mathrm{v}_{\mathrm{i}}\right)+\mathrm{f}\left(\mathrm{v}_{\mathrm{i}}\right)=2 \mathrm{i}+\frac{5}{2} \mathrm{n}+3-\mathrm{i}+2 \mathrm{n}+3-\mathrm{i}=\frac{9}{2} \mathrm{n}+6=\lambda_{3}$.

For $\frac{\mathrm{n}}{2}+1 \leq \mathrm{i} \leq \mathrm{n}, \mathrm{f}\left(\mathrm{u}_{\mathrm{i}}\right)+\mathrm{f}\left(\mathrm{u}_{\mathrm{i}} \mathrm{v}_{\mathrm{i}}\right)+\mathrm{f}\left(\mathrm{v}_{\mathrm{i}}\right)=2 \mathrm{i}-\mathrm{n}+1+\frac{7}{2} \mathrm{n}+2-\mathrm{i}+2 \mathrm{n}+3-\mathrm{i}=\frac{9}{2} \mathrm{n}+6=\lambda_{3}$.

Hence for each edge $u v \in E, f(u)+f(u v)+f(v)$ yields any one of the magic constants $\lambda_{1}=6(n+1), \lambda_{2}=5 n+6$ and $\lambda_{3}=\frac{9}{2} n+6$. Hence the Book $B_{n}$ is an edge trimagic total labeling when $\mathrm{n}$ is even.

Case 2. $\mathrm{n}$ is odd. 
Edge Trimagic Total Labeling of Mobius Ladder, Book and Dragon Graphs

Define a bijection $\mathrm{f}: \mathrm{V} \cup \mathrm{E} \rightarrow\{1,2, \ldots, 5 \mathrm{n}+3\}$ such that

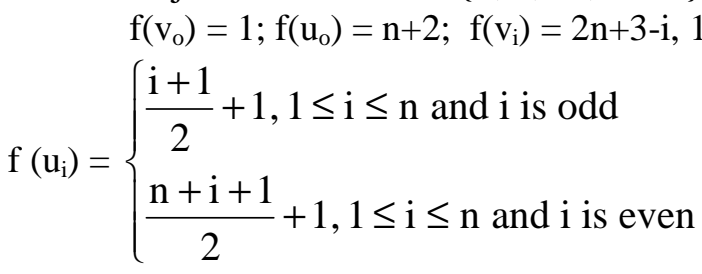

$\mathrm{f}\left(\mathrm{v}_{\mathrm{o}} \mathrm{v}_{\mathrm{i}}\right)=4 \mathrm{n}+\mathrm{i}+2,1 \leq \mathrm{i} \leq \mathrm{n} ;$

$f\left(u_{o} u_{i}\right)=\left\{\begin{array}{l}4 n+3-\frac{i+1}{2}, 1 \leq \mathrm{i} \leq \mathrm{n} \text { and } \mathrm{i} \text { is odd } \\ 3 \mathrm{n}+\frac{\mathrm{n}}{2}+2+\frac{1-\mathrm{i}}{2}, 1 \leq \mathrm{i} \leq \mathrm{n} \text { and } \mathrm{i} \text { is even }\end{array}\right.$

$\mathrm{f}\left(\mathrm{u}_{\mathrm{i}} \mathrm{v}_{\mathrm{i}}\right)=\left\{\begin{array}{l}2 \mathrm{n}+\frac{\mathrm{n}}{2}+2+\frac{\mathrm{i}}{2}, 1 \leq \mathrm{i} \leq \mathrm{n} \text { and } \mathrm{i} \text { is odd } \\ 2 \mathrm{n}+2+\frac{\mathrm{i}}{2}, 1 \leq \mathrm{i} \leq \mathrm{n} \text { and } \mathrm{i} \text { is even }\end{array}\right.$

and $\mathrm{f}\left(\mathrm{u}_{\mathrm{o}} \mathrm{v}_{\mathrm{o}}\right)=5 \mathrm{n}+3$.

To prove this labeling is an edge trimagic total labeling.

For the edges $v_{0} v_{i}, 1 \leq i \leq n, f\left(v_{0}\right)+f\left(v_{0} v_{i}\right) f\left(v_{i}\right)=1+4 n+i+2+2 n+3-i=6(n+1)=\lambda_{1}$.

For the edge $v_{0} u_{o}, f\left(v_{o}\right)+f\left(v_{o} u_{o}\right)+f\left(u_{o}\right)=1+5 n+3+n+2=6(n+1)=\lambda_{1}$.

Consider the edges $\mathrm{u}_{0} \mathrm{u}_{\mathrm{i}}, 1 \leq \mathrm{i} \leq \mathrm{n}$.

For odd $i, f\left(u_{0}\right)+f\left(u_{0} u_{i}\right)+f\left(u_{i}\right)=n+2+4 n+3-\left(\frac{i+1}{2}\right)+\left(\frac{i+1}{2}\right)+1=5 n+6=\lambda_{2}$.

For even $\mathrm{i}, \mathrm{f}\left(\mathrm{u}_{\mathrm{o}}\right)+\mathrm{f}\left(\mathrm{u}_{\mathrm{o}} \mathrm{u}_{\mathrm{i}}\right)+\mathrm{f}\left(\mathrm{u}_{\mathrm{i}}\right)=\mathrm{n}+2+3 \mathrm{n}+\frac{\mathrm{n}}{2}+2+\frac{1-\mathrm{i}}{2}+\frac{\mathrm{n}+\mathrm{i}+1}{2}+1=5 \mathrm{n}+6=\lambda_{2}$.

Consider the edges $\mathrm{u}_{\mathrm{i}} \mathrm{v}_{\mathrm{i}}, 1 \leq \mathrm{i} \leq \mathrm{n}$,

For odd $\mathrm{i}, \mathrm{f}\left(\mathrm{u}_{\mathrm{i}}\right)+\mathrm{f}\left(\mathrm{u}_{\mathrm{i}} \mathrm{v}_{\mathrm{i}}\right)+\mathrm{f}\left(\mathrm{v}_{\mathrm{i}}\right)=\frac{\mathrm{i}+1}{2}+1+2 \mathrm{n}+\frac{\mathrm{n}}{2}+2+\frac{\mathrm{i}}{2}+2 \mathrm{n}+3-\mathrm{i}=\frac{9 \mathrm{n}+13}{2}=\lambda_{3}$.

For even i, $\mathrm{f}\left(\mathrm{u}_{\mathrm{i}}\right)+\mathrm{f}\left(\mathrm{u}_{\mathrm{i}} \mathrm{v}_{\mathrm{i}}\right)+\mathrm{f}\left(\mathrm{v}_{\mathrm{i}}\right)=\frac{\mathrm{n}+3+\mathrm{i}}{2}+2(\mathrm{n}+1)+\frac{\mathrm{i}}{2}+2 \mathrm{n}+3-\mathrm{i}=\frac{9 \mathrm{n}+13}{2}=\lambda_{3}$.

Hence for each edge $u v \in E, f(u)+f(u v)+f(v)$ yields any one of the magic constants $\lambda_{1}=6(n+1), \lambda_{2}=5 n+6$ and $\lambda_{3}=\frac{9 n+13}{2}$. Therefore, the Book $B_{n}$ is an edge trimagic total labeling when $\mathrm{n}$ is odd. From cases (1) and (2), the Book $\mathrm{B}_{\mathrm{n}}$ is an edge trimagic total labeling.

Corollary 2.5. The Book $B_{n}$ is a supper edge trimagic total labeling graph.

Proof: We proved that the Book $B_{n}$ is an edge trimagic total labeling with $2 n+2$ vertices. The labeling given in the proof of Theorem 2.4 is as follows:

For even $\mathrm{n}, \mathrm{f}\left(\mathrm{v}_{\mathrm{o}}\right)=1 ; \mathrm{f}\left(\mathrm{u}_{\mathrm{o}}\right)=\mathrm{n}+2 ; \mathrm{f}\left(\mathrm{v}_{\mathrm{i}}\right)=2 \mathrm{n}+3-\mathrm{i}, 1 \leq \mathrm{i} \leq \mathrm{n}$ and 


\section{Jayasekaran and J. Little Flower}

$$
\mathrm{f}\left(\mathrm{u}_{\mathrm{i}}\right)=\left\{\begin{array}{l}
2 \mathrm{i}, \quad 1 \leq \mathrm{i} \leq \frac{\mathrm{n}}{2} \\
2 \mathrm{i}-\mathrm{n}+1, \frac{\mathrm{n}}{2}+1 \leq \mathrm{i} \leq \mathrm{n}
\end{array}\right.
$$

For odd $\mathrm{n}, \mathrm{f}\left(\mathrm{v}_{0}\right)=1 ; \mathrm{f}\left(\mathrm{u}_{0}\right)=\mathrm{n}+2 ; \mathrm{f}\left(\mathrm{v}_{\mathrm{i}}\right)=2 \mathrm{n}+3-\mathrm{i}, 1 \leq \mathrm{i} \leq \mathrm{n}$ and

$$
f\left(u_{i}\right)=\left\{\begin{array}{l}
\frac{i+1}{2}+1, \quad 1 \leq \mathrm{i} \leq \mathrm{n} \text { and } \mathrm{i} \text { is odd } \\
\frac{\mathrm{n}+\mathrm{i}+1}{2}+1, \quad 1 \leq \mathrm{i} \leq \mathrm{n} \text { and } \mathrm{i} \text { is even }
\end{array}\right.
$$

Hence, the $2 n+2$ vertices get labels $1,2, \ldots, 2 n+2$. Therefore, the Book $B_{n}$ is super edge trimagic total labeling graph.

Example 2.6. An edge trimagic total labeling of the Book $B_{10}$ and $B_{7}$ are given in figure 2 and figure 3 , respectively.

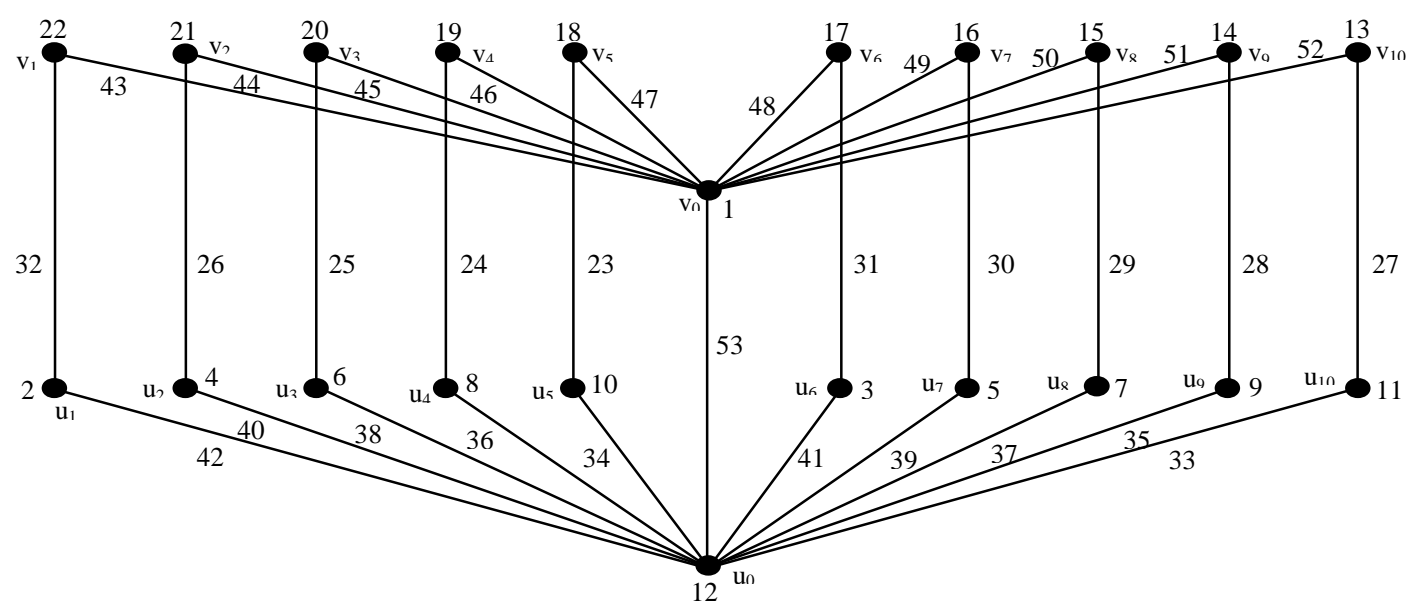

Figure 2: $\mathrm{B}_{10}\left(\mathrm{~S}_{10} \times \mathrm{P}_{2}\right)$ with $\lambda_{1}=66, \lambda_{2}=56$ and $\lambda_{3}=51$

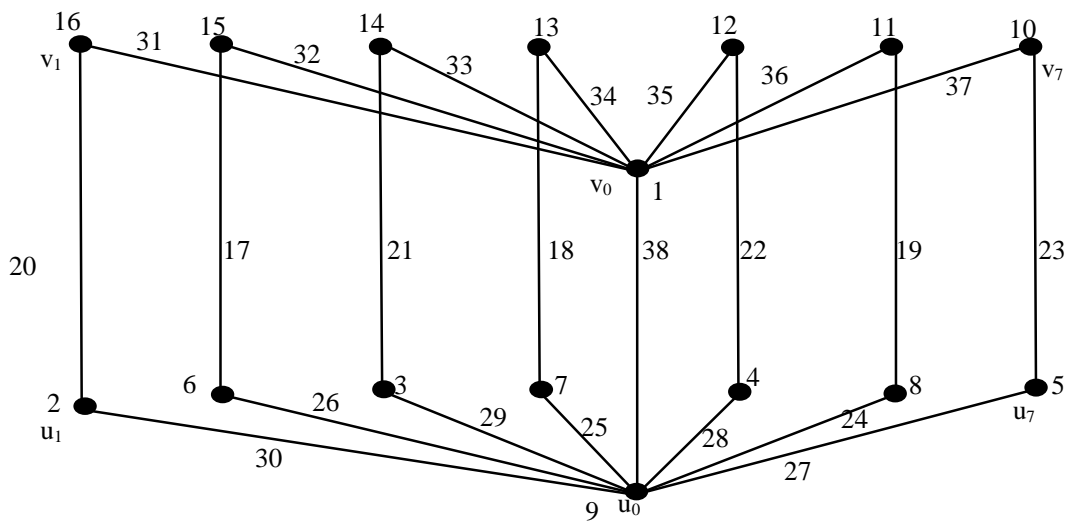

Figure 3: $B_{7}\left(S_{7} \times P_{2}\right)$ with $\lambda_{1}=48, \lambda_{2}=41$ and $\lambda_{3}=38$ 
Edge Trimagic Total Labeling of Mobius Ladder, Book and Dragon Graphs

Theorem 2.7. The (n, $l)$ - dragon graph is an edge trimagic total labeling.

Proof: Let $\mathrm{V}=\left\{\mathrm{v}_{\mathrm{i}} / 1 \leq \mathrm{i} \leq l\right\} \cup\left\{\mathrm{u}_{\mathrm{j}} / 1 \leq \mathrm{j} \leq \mathrm{n}\right\}$ be the vertex set and $\mathrm{E}=\left\{\mathrm{v}_{\mathrm{i}} \mathrm{v}_{\mathrm{i}+1} /\right.$ $1 \leq \mathrm{i} \leq l-1\} \cup\left\{\mathrm{u}_{1} \mathrm{v}_{1}, \mathrm{u}_{1} \mathrm{u}_{\mathrm{n}}\right\} \cup\left\{\mathrm{u}_{\mathrm{j}} \mathrm{u}_{\mathrm{j}+1} / 1 \leq \mathrm{j} \leq \mathrm{n}-1\right\}$ be the edge set.

Then $(\mathrm{n}, l)-$ dragon has $\mathrm{n}+l$ vertices and $\mathrm{n}+l$ edges.

Case 1 . Both $\mathrm{n}$ and $l$ are even.

Define a bijection $\mathrm{f}: \mathrm{V} \cup \mathrm{E} \rightarrow\{1,2, \ldots, 2(\mathrm{n}+l)\}$ such that

$\mathrm{f}\left(\mathrm{v}_{\mathrm{i}}\right)=\left\{\begin{array}{l}\frac{l+\mathrm{i}+3}{2}, \quad \mathrm{i} \text { is odd and } 1 \leq \mathrm{i} \leq l \\ \frac{\mathrm{i}+2}{2}, \quad \mathrm{i} \text { is even and } 1 \leq \mathrm{i} \leq l\end{array}\right.$

$\mathrm{f}\left(\mathrm{u}_{\mathrm{j}}\right)=l+\mathrm{j}, 2 \leq \mathrm{j} \leq \mathrm{n} ; \mathrm{f}\left(\mathrm{u}_{1}\right)=1 ; \mathrm{f}\left(\mathrm{v}_{\mathrm{i}} \mathrm{v}_{\mathrm{i}+1}\right)=2(\mathrm{n}+l-1)-\mathrm{i}, 1 \leq \mathrm{i} \leq l-1 ; \mathrm{f}\left(\mathrm{u}_{1} \mathrm{u}_{2}\right)=2(\mathrm{n}+l)-$ $1 ; \mathrm{f}\left(\mathrm{u}_{1} \mathrm{u}_{\mathrm{n}}\right)=2(\mathrm{n}+l) ; \mathrm{f}\left(\mathrm{u}_{1} \mathrm{v}_{1}\right)=2(\mathrm{n}+l-1)$ and

$f\left(u_{j} u_{j-1}\right)= \begin{cases}2 \mathrm{n}+l-2 \mathrm{j}+3, & 2<\mathrm{j} \leq \frac{\mathrm{n}}{2}+1 \\ 3 \mathrm{n}+l-2 \mathrm{j}+2, & \frac{\mathrm{n}}{2}+1<\mathrm{j} \leq \mathrm{n}\end{cases}$

To prove this labeling is an edge trimagic total labeling.

Consider the edges $\mathrm{v}_{\mathrm{i}} \mathrm{v}_{\mathrm{i}+1}, 1 \leq \mathrm{i} \leq l-1$.

For odd $\mathrm{i}, \mathrm{f}\left(\mathrm{v}_{\mathrm{i}}\right)+\mathrm{f}\left(\mathrm{v}_{\mathrm{i}} \mathrm{v}_{\mathrm{i}+1}\right)+\mathrm{f}\left(\mathrm{v}_{\mathrm{i}+1}\right)=\frac{l+\mathrm{i}+3}{2}+2(\mathrm{n}+l-1)-\mathrm{i}+\frac{\mathrm{i}+3}{2}=2 \mathrm{n}+\frac{5}{2} l+1=\lambda_{1}$.

For even $\mathrm{i}, \mathrm{f}\left(\mathrm{v}_{\mathrm{i}}\right)+\mathrm{f}\left(\mathrm{v}_{\mathrm{i}} \mathrm{v}_{\mathrm{i}+1}\right)+\mathrm{f}\left(\mathrm{v}_{\mathrm{i}+1}\right)=\frac{\mathrm{i}+2}{2}+2(\mathrm{n}+l-1)-\mathrm{i}+\frac{l+\mathrm{i}+4}{2}=2 \mathrm{n}+\frac{5}{2} l+1=\lambda_{1}$.

For the edge $\mathrm{u}_{1} \mathrm{v}_{1}, \mathrm{f}\left(\mathrm{u}_{1}\right)+\mathrm{f}\left(\mathrm{u}_{1} \mathrm{v}_{1}\right)+\mathrm{f}\left(\mathrm{v}_{1}\right)=1+2(\mathrm{n}+l-1)+\frac{l+4}{2}=2 \mathrm{n}+\frac{5}{2} l+1=\lambda_{1}$.

For the edge $u_{1} u_{2}, f\left(u_{1}\right)+f\left(u_{1} u_{2}\right)+f\left(u_{2}\right)=1+2(n+l)-1+l+2=2 n+3 l+2=\lambda_{2}$.

For the edges $u_{j} u_{j-1}, 2<j \leq \frac{n}{2}+1, f\left(u_{j}\right)+f\left(u_{j} u_{j-1}\right)+f\left(u_{j-1}\right)=l+j+2 n+l-2 j+3+l+j-1$

$=2 \mathrm{n}+3 l+2=\lambda_{2}$.

For the edge $\mathrm{u}_{1} \mathrm{u}_{\mathrm{n}}, \mathrm{f}\left(\mathrm{u}_{1}\right)+\mathrm{f}\left(\mathrm{u}_{1} \mathrm{u}_{\mathrm{n}}\right)+\mathrm{f}\left(\mathrm{u}_{\mathrm{n}}\right)=1+2(\mathrm{n}+l)+l+\mathrm{n}=3(\mathrm{n}+l)+1=\lambda_{3}$.

For the edges $u_{j} u_{j-1}, \frac{n}{2}+1<j \leq n, f\left(u_{j}\right)+f\left(u_{j} u_{j-1}\right)+f\left(u_{j-1}\right)=l+j+3 n+l-2 j+2+l+j-1=$ $3(\mathrm{n}+l)+1=\lambda_{3}$.

Hence for each edge $u v \in E, f(u)+f(u v)+f(v)$ yields any one of the magic constants $\lambda_{1}=2 \mathrm{n}+\frac{5}{2} l+1, \lambda_{2}=2 \mathrm{n}+3 l+2$ and $\lambda_{3}=3(\mathrm{n}+l)+1$. Therefore, the (n, $\left.l\right)-$ dragon is an edge trimagic total labeling when both $\mathrm{n}$ and $l$ are even.

Case 2. $\mathrm{n}$ is even and $l$ is odd.

Define a bijection $\mathrm{f}: \mathrm{V} \cup \mathrm{E} \rightarrow\{1,2, \ldots, 2(\mathrm{n}+l)\}$ such that 


\section{Jayasekaran and J. Little Flower}

$\mathrm{f}\left(\mathrm{v}_{\mathrm{i}}\right)= \begin{cases}\frac{l+\mathrm{i}+2}{2}, & \mathrm{i} \text { is odd } \text { and } 1 \leq \mathrm{i} \leq l \\ \frac{\mathrm{i}+2}{2}, & \mathrm{i} \text { is even and } 1 \leq \mathrm{i} \leq l\end{cases}$

$\mathrm{f}\left(\mathrm{u}_{\mathrm{j}}\right)=l+\mathrm{j}, 2 \leq \mathrm{j} \leq \mathrm{n} ; \mathrm{f}\left(\mathrm{u}_{1}\right)=1 ; \mathrm{f}\left(\mathrm{v}_{\mathrm{i}} \mathrm{v}_{\mathrm{i}+1}\right)=2(\mathrm{n}+l-1)-\mathrm{i}, 1 \leq \mathrm{i} \leq l-1 ; \mathrm{f}\left(\mathrm{u}_{1} \mathrm{u}_{2}\right)=2(\mathrm{n}+l)-1 ; \mathrm{f}\left(\mathrm{u}_{1} \mathrm{u}_{\mathrm{n}}\right)$ $=2(\mathrm{n}+l) ; \mathrm{f}\left(\mathrm{u}_{1} \mathrm{v}_{1}\right)=2(\mathrm{n}+l-1)$ and

$$
f\left(u_{j} u_{j-1}\right)= \begin{cases}2 \mathrm{n}+l-2 \mathrm{j}+3, & 2<\mathrm{j} \leq \frac{\mathrm{n}}{2}+1 \\ 3 \mathrm{n}+l-2 \mathrm{j}+2, & \frac{\mathrm{n}}{2}+1<\mathrm{j} \leq \mathrm{n}\end{cases}
$$

To prove this labeling is an edge trimagic total labeling.

Consider the edges $\mathrm{v}_{\mathrm{i}} \mathrm{v}_{\mathrm{i}+1}, 1 \leq \mathrm{i} \leq l-1$.

For odd $\mathrm{i}, \mathrm{f}\left(\mathrm{v}_{\mathrm{i}}\right)+\mathrm{f}\left(\mathrm{v}_{\mathrm{i}} \mathrm{v}_{\mathrm{i}+1}\right)+\mathrm{f}\left(\mathrm{v}_{\mathrm{i}+1}\right)=\frac{l+\mathrm{i}+2}{2}+2(\mathrm{n}+l-1)-\mathrm{i}+\frac{\mathrm{i}+3}{2}=2(\mathrm{n}+l)+\frac{l+1}{2}=\lambda_{1}$.

For even $\mathrm{i}, \mathrm{f}\left(\mathrm{v}_{\mathrm{i}}\right)+\mathrm{f}\left(\mathrm{v}_{\mathrm{i}} \mathrm{v}_{\mathrm{i}+1}\right)+\mathrm{f}\left(\mathrm{v}_{\mathrm{i}+1}\right)=\frac{\mathrm{i}+2}{2}+2(\mathrm{n}+l-1)-\mathrm{i}+\frac{l+\mathrm{i}+3}{2}=2(\mathrm{n}+l)+\frac{l+1}{2}=\lambda_{1}$.

For the edge $\mathrm{u}_{1} \mathrm{v}_{1}, \mathrm{f}\left(\mathrm{u}_{1}\right)+\mathrm{f}\left(\mathrm{u}_{1} \mathrm{v}_{1}\right)+\mathrm{f}\left(\mathrm{v}_{1}\right)=1+2(\mathrm{n}+l-1)+\frac{l+3}{2}=2(\mathrm{n}+l)+\frac{l+1}{2}=\lambda_{1}$.

For the edge $u_{1} u_{2}, f\left(u_{1}\right)+f\left(u_{1} u_{2}\right)+f\left(u_{2}\right)=1+2(n+l)-1+l+2=2 n+3 l+2=\lambda_{2}$.

For the edge $\mathrm{u}_{1} \mathrm{u}_{\mathrm{n}}, \mathrm{f}\left(\mathrm{u}_{1}\right)+\mathrm{f}\left(\mathrm{u}_{1} \mathrm{u}_{\mathrm{n}}\right)+\mathrm{f}\left(\mathrm{u}_{\mathrm{n}}\right)=1+2(\mathrm{n}+l)+l+\mathrm{n}=3(\mathrm{n}+l)+1=\lambda_{3}$.

For the edges $u_{j} u_{j-1}, \quad 2<j \leq \frac{n}{2}+1, \quad f\left(u_{j}\right)+f\left(u_{j} u_{j-1}\right)+f\left(u_{j-1}\right)=l+j+2 n+l-2 j+3+l+j-1$ $=2 \mathrm{n}+3 l+2=\lambda_{2}$.

For the edges $\mathrm{u}_{\mathrm{j}} \mathrm{u}_{\mathrm{j}-1}, \frac{\mathrm{n}}{2}+1<\mathrm{j} \leq \mathrm{n}, \mathrm{f}\left(\mathrm{u}_{\mathrm{j}}\right)+\mathrm{f}\left(\mathrm{u}_{\mathrm{j}} \mathrm{u}_{\mathrm{j}-1}\right)+\mathrm{f}\left(\mathrm{u}_{\mathrm{j}-1}\right)=l+\mathrm{j}+3 \mathrm{n}+l-2 \mathrm{j}+2+l+\mathrm{j}-1$ $=3(\mathrm{n}+l)+1=\lambda_{3}$.

Hence for each edge $u v \in E, f(u)+f(u v)+f(v)$ yields any one of the magic constants $\lambda_{1}=$ $2(\mathrm{n}+l)+\frac{l+1}{2}, \lambda_{2}=2 \mathrm{n}+3 l+2$ and $\lambda_{3}=3(\mathrm{n}+l)+1$. Therefore, the (n, $\left.l\right)-$ dragon is an edge trimagic total labeling when $\mathrm{n}$ is even and $l$ is odd.

Case 3. Both $\mathrm{n}$ and $l$ are odd.

Define a bijection $\mathrm{f}: \mathrm{V} \cup \mathrm{E} \rightarrow\{1,2, \ldots, 2(\mathrm{n}+l)\}$ such that

$\mathrm{f}\left(\mathrm{v}_{\mathrm{i}}\right)= \begin{cases}\frac{l+\mathrm{i}+2}{2}, & \mathrm{i} \text { is odd and } 1 \leq \mathrm{i} \leq l \\ \frac{\mathrm{i}+2}{2}, & \mathrm{i} \text { is even and } 1 \leq \mathrm{i} \leq l\end{cases}$ 
Edge Trimagic Total Labeling of Mobius Ladder, Book and Dragon Graphs $\mathrm{f}\left(\mathrm{u}_{\mathrm{j}}\right)=\left\{\begin{array}{l}l+\frac{\mathrm{j}+1}{2}, 2 \leq \mathrm{j} \leq \mathrm{n} \text { and } \mathrm{j} \text { is odd } \\ l+\frac{\mathrm{n}+\mathrm{j}+1}{2}, 2 \leq \mathrm{j} \leq \mathrm{n} \text { and } \mathrm{j} \text { is even }\end{array}\right.$

$\mathrm{f}\left(\mathrm{u}_{1}\right)=1 ; \mathrm{f}\left(\mathrm{v}_{\mathrm{i}} \mathrm{v}_{\mathrm{i}+1}\right)=2(\mathrm{n}+l)-\mathrm{i}, 1 \leq \mathrm{i} \leq l-1 ; \mathrm{f}\left(\mathrm{u}_{1} \mathrm{v}_{1}\right)=2(\mathrm{n}+l) ; \mathrm{f}\left(\mathrm{u}_{1} \mathrm{u}_{2}\right)=\mathrm{n}+l+1 ; \mathrm{f}\left(\mathrm{u}_{1} \mathrm{u}_{\mathrm{n}}\right)=\mathrm{n}+l+2$ and $\mathrm{f}\left(\mathrm{u}_{\mathrm{j}} \mathrm{u}_{\mathrm{j}+1}\right)=2 \mathrm{n}+l+2-\mathrm{j}, 2 \leq \mathrm{j} \leq \mathrm{n}-1$.

To prove this labeling is an edge trimagic total labeling.

Consider the edges $\mathrm{v}_{\mathrm{i}} \mathrm{v}_{\mathrm{i}+1}, 1 \leq \mathrm{i} \leq l-1$.

For odd $\mathrm{i}, \mathrm{f}\left(\mathrm{v}_{\mathrm{i}}\right)+\mathrm{f}\left(\mathrm{v}_{\mathrm{i}} \mathrm{v}_{\mathrm{i}+1}\right)+\mathrm{f}\left(\mathrm{v}_{\mathrm{i}+1}\right)=\frac{l+\mathrm{i}+2}{2}+2(\mathrm{n}+l)-\mathrm{i}+\frac{\mathrm{i}+3}{2}=2(\mathrm{n}+l+1)+\frac{l+1}{2}=\lambda_{1}$.

For even $\mathrm{i}, \mathrm{f}\left(\mathrm{v}_{\mathrm{i}}\right)+\mathrm{f}\left(\mathrm{v}_{\mathrm{i}} \mathrm{v}_{\mathrm{i}+1}\right)+\mathrm{f}\left(\mathrm{v}_{\mathrm{i}+1}\right)=\frac{\mathrm{i}+2}{2}+2(\mathrm{n}+l)-\mathrm{i}+\frac{l+\mathrm{i}+3}{2}=2(\mathrm{n}+l+1)+\frac{l+1}{2}=\lambda_{1}$.

For the edge $\mathrm{u}_{1} \mathrm{v}_{1}, \mathrm{f}\left(\mathrm{u}_{1}\right)+\mathrm{f}\left(\mathrm{u}_{1} \mathrm{v}_{1}\right)+\mathrm{f}\left(\mathrm{v}_{1}\right)=1+2(\mathrm{n}+l)+\frac{l+3}{2}=2(\mathrm{n}+l+1)+\frac{l+1}{2}=\lambda_{1}$.

For the edge $\mathrm{u}_{1} \mathrm{u}_{2}, \mathrm{f}\left(\mathrm{u}_{1}\right)+\mathrm{f}\left(\mathrm{u}_{1} \mathrm{u}_{2}\right)+\mathrm{f}\left(\mathrm{u}_{2}\right)=1+\mathrm{n}+l+1+l+\frac{\mathrm{n}+3}{2}=\mathrm{n}+2 l+3+\frac{\mathrm{n}+1}{2}=\lambda_{2}$.

For the edge $\mathrm{u}_{1} \mathrm{u}_{\mathrm{n}}, \mathrm{f}\left(\mathrm{u}_{1}\right)+\mathrm{f}\left(\mathrm{u}_{1} \mathrm{u}_{\mathrm{n}}\right)+\mathrm{f}\left(\mathrm{u}_{\mathrm{n}}\right)=1+\mathrm{n}+l+2+l+\frac{\mathrm{n}+1}{2}=\mathrm{n}+2 l+3+\frac{\mathrm{n}+1}{2}=\lambda_{2}$.

Consider the edges $\mathrm{u}_{\mathrm{j}} \mathrm{u}_{\mathrm{j}+1}, 2 \leq \mathrm{j} \leq \mathrm{n}-1$.

For odd j,

$\mathrm{f}\left(\mathrm{u}_{\mathrm{j}}\right)+\mathrm{f}\left(\mathrm{u}_{\mathrm{j}} \mathrm{u}_{\mathrm{j}+1}\right)+\mathrm{f}\left(\mathrm{u}_{\mathrm{j}+1}\right)=l+\frac{\mathrm{j}+1}{2}+2 \mathrm{n}+l+2-\mathrm{j}+l+\frac{\mathrm{n}+\mathrm{j}+2}{2}=2 \mathrm{n}+3 l+3+\frac{\mathrm{n}+1}{2}=\lambda_{3}$.

For even $\mathrm{j}$,

$\mathrm{f}\left(\mathrm{u}_{\mathrm{j}}\right)+\mathrm{f}\left(\mathrm{u}_{\mathrm{j}} \mathrm{u}_{\mathrm{j}+1}\right)+\mathrm{f}\left(\mathrm{u}_{\mathrm{j}+1}\right)=l+\frac{\mathrm{n}+\mathrm{j}+1}{2}+2 \mathrm{n}+l+2-\mathrm{j}+l+\frac{\mathrm{j}+2}{2}=2 \mathrm{n}+3 l+3+\frac{\mathrm{n}+1}{2}=\lambda_{3}$.

Hence, for each edge $u v \in E, f(u)+f(u v)+f(v)$ yields any one of the magic constants $\lambda_{1}=$ $2(\mathrm{n}+l+1)+\frac{l+1}{2} ; \lambda_{2}=\mathrm{n}+2 l+3+\frac{\mathrm{n}+1}{2}$ and $\lambda_{3}=2 \mathrm{n}+3 l+3+\frac{\mathrm{n}+1}{2}$. Therefore, the $(\mathrm{n}, l)-$ dragon is an edge trimagic total labeling when $\mathrm{n}$ is odd and $l$ is odd. Case $4 . \mathrm{n}$ is odd and $l$ is even.

Define a bijection $\mathrm{f}: \mathrm{V} \cup \mathrm{E} \rightarrow\{1,2, \ldots, 2(\mathrm{n}+l)\}$ such that

$\mathrm{f}\left(\mathrm{v}_{\mathrm{i}}\right)= \begin{cases}\frac{l+\mathrm{i}+3}{2}, & 1 \leq \mathrm{i} \leq \mathrm{n}, \mathrm{i} \text { is odd } \\ \frac{\mathrm{i}+2}{2}, & 1 \leq \mathrm{i} \leq \mathrm{n}, \mathrm{i} \text { is even }\end{cases}$

$\mathrm{f}\left(\mathrm{u}_{\mathrm{j}}\right)=\left\{\begin{array}{l}l+\frac{\mathrm{j}+1}{2}, 2 \leq \mathrm{j} \leq \mathrm{n}, \mathrm{j} \text { is odd } \\ l+\frac{\mathrm{n}+\mathrm{j}+1}{2}, 2 \leq \mathrm{j} \leq \mathrm{n}, \mathrm{j} \text { is even }\end{array}\right.$ 


\section{Jayasekaran and J. Little Flower}

$\mathrm{f}\left(\mathrm{u}_{1}\right)=1 ; \mathrm{f}\left(\mathrm{v}_{\mathrm{i}} \mathrm{v}_{\mathrm{i}+1}\right)=2(\mathrm{n}+l)-\mathrm{i}, 1 \leq \mathrm{i} \leq l-1 ; \mathrm{f}\left(\mathrm{u}_{1} \mathrm{v}_{1}\right)=2(\mathrm{n}+l) ; \mathrm{f}\left(\mathrm{u}_{1} \mathrm{u}_{2}\right)=\mathrm{n}+l+1 ; \mathrm{f}\left(\mathrm{u}_{1} \mathrm{u}_{\mathrm{n}}\right)=\mathrm{n}+l+2 ;$ $\mathrm{f}\left(\mathrm{u}_{\mathrm{j}} \mathrm{u}_{\mathrm{j}+1}\right)=2 \mathrm{n}+l+2-\mathrm{j}, 2 \leq \mathrm{j} \leq \mathrm{n}-1$.

To prove this labeling is an edge trimagic total labeling.

Consider the edges $\mathrm{v}_{\mathrm{i}} \mathrm{v}_{\mathrm{i}+1}, 1 \leq \mathrm{i} \leq l-1$.

For odd $\mathrm{i}, \mathrm{f}\left(\mathrm{v}_{\mathrm{i}}\right)+\mathrm{f}\left(\mathrm{v}_{\mathrm{i}} \mathrm{v}_{\mathrm{i}+1}\right)+\mathrm{f}\left(\mathrm{v}_{\mathrm{i}+1}\right)=\frac{l+\mathrm{i}+3}{2}+2(\mathrm{n}+l)-\mathrm{i}+\frac{\mathrm{i}+3}{2}=2(\mathrm{n}+l)+3+\frac{l}{2}=\lambda_{1}$.

For even $\mathrm{i}, \mathrm{f}\left(\mathrm{v}_{\mathrm{i}}\right)+\mathrm{f}\left(\mathrm{v}_{\mathrm{i}} \mathrm{v}_{\mathrm{i}+1}\right)+\mathrm{f}\left(\mathrm{v}_{\mathrm{i}+1}\right)=\frac{\mathrm{i}+2}{2}+2(\mathrm{n}+l)-\mathrm{i}+\frac{l+\mathrm{i}+4}{2}=2(\mathrm{n}+l)+3+\frac{l}{2}=\lambda_{1}$.

For the edge $\mathrm{u}_{1} \mathrm{v}_{1}, \mathrm{f}\left(\mathrm{u}_{1}\right)+\mathrm{f}\left(\mathrm{u}_{1} \mathrm{v}_{1}\right)+\mathrm{f}\left(\mathrm{v}_{1}\right)=1+2(\mathrm{n}+l)+\frac{l+4}{2}=2(\mathrm{n}+l)+3+\frac{l}{2}=\lambda_{1}$.

For the edge $u_{1} u_{2}, f\left(u_{1}\right)+f\left(u_{1} u_{2}\right)+f\left(u_{2}\right)=1+n+l+1+\frac{n+2 l+3}{2}=n+2 l+3+\frac{n+1}{2}=\lambda_{2}$.

For the edge $u_{1} u_{n}, f\left(u_{1}\right)+f\left(u_{1} u_{n}\right)+f\left(u_{n}\right)=1+n+l+2+\frac{n+2 l+1}{2}=n+2 l+3+\frac{n+1}{2}=\lambda_{2}$.

Consider the edges $\mathrm{u}_{\mathrm{j}} \mathrm{u}_{\mathrm{j}+1}, 2 \leq \mathrm{j} \leq \mathrm{n}-1$.

For odd $\mathrm{j}$,

$\mathrm{f}\left(\mathrm{u}_{\mathrm{j}}\right)+\mathrm{f}\left(\mathrm{u}_{\mathrm{j}} \mathrm{u}_{\mathrm{j}+1}\right)+\mathrm{f}\left(\mathrm{u}_{\mathrm{j}+1}\right)=l+\frac{\mathrm{j}+1}{2}+2 \mathrm{n}+l+2-\mathrm{j}+l+\frac{\mathrm{n}+\mathrm{j}+2}{2}=2 \mathrm{n}+3 l+3+\frac{\mathrm{n}+1}{2}=\lambda_{3}$.

For even $\mathrm{j}$,

$\mathrm{f}\left(\mathrm{u}_{\mathrm{j}}\right)+\mathrm{f}\left(\mathrm{u}_{\mathrm{j}} \mathrm{u}_{\mathrm{j}+1}\right)+\mathrm{f}\left(\mathrm{u}_{\mathrm{j}+1}\right)=l+\frac{\mathrm{n}+\mathrm{j}+1}{2}+2 \mathrm{n}+l+2-\mathrm{j}+l+\frac{\mathrm{j}+2}{2}=2 \mathrm{n}+3 l+3+\frac{\mathrm{n}+1}{2}=\lambda_{3}$.

Hence for each edge $u v \in E, f(u)+f(u v)+f(v)$ yields any one of the magic constants $\lambda_{1}=$ $2(\mathrm{n}+l)+3+\frac{l}{2} ; \lambda_{2}=\mathrm{n}+2 l+3+\frac{\mathrm{n}+1}{2}$ and $\lambda_{3}=2 \mathrm{n}+3 l+3+\frac{\mathrm{n}+1}{2}$. Therefore, the $(\mathrm{n}, l)$ - dragon is an edge trimagic total labeling when $\mathrm{n}$ is odd and $l$ is even.

Corollary 2.8. The $(\mathrm{n}, l)$ - dragon graph is super edge trimagic total labeling.

Proof: We proved that the $(\mathrm{n}, l)-$ dragon is an edge trimagic total labeling with $\mathrm{n}+l$ vertices. The labeling given in the proof of Theorem 2.7 is as follows:

For even $\mathrm{n}$ and even $l$,

$\mathrm{f}\left(\mathrm{v}_{\mathrm{i}}\right)=\left\{\begin{array}{l}\frac{l+\mathrm{i}+3}{2}, \quad \mathrm{i} \text { is odd } \text { and } 1 \leq \mathrm{i} \leq l \\ \frac{\mathrm{i}+2}{2}, \quad \mathrm{i} \text { is even and } 1 \leq \mathrm{i} \leq l\end{array}\right.$

$\mathrm{f}\left(\mathrm{u}_{\mathrm{j}}\right)=l+\mathrm{j}, 2 \leq \mathrm{j} \leq \mathrm{n} ; \mathrm{f}\left(\mathrm{u}_{1}\right)=1$.

For even $\mathrm{n}$ and odd $l$,

$\mathrm{f}\left(\mathrm{v}_{\mathrm{i}}\right)= \begin{cases}\frac{l+\mathrm{i}+2}{2}, & \mathrm{i} \text { is odd } \text { and } 1 \leq \mathrm{i} \leq l \\ \frac{\mathrm{i}+2}{2}, & \mathrm{i} \text { is even } \text { and } 1 \leq \mathrm{i} \leq l\end{cases}$

$\mathrm{f}\left(\mathrm{u}_{\mathrm{j}}\right)=l+\mathrm{j}, 2 \leq \mathrm{j} \leq \mathrm{n} ; \mathrm{f}\left(\mathrm{u}_{1}\right)=1$. 
Edge Trimagic Total Labeling of Mobius Ladder, Book and Dragon Graphs For odd $\mathrm{n}$ and odd $l$,

$$
\begin{aligned}
& \mathrm{f}\left(\mathrm{v}_{\mathrm{i}}\right)=\left\{\begin{array}{l}
\frac{l+\mathrm{i}+2}{2}, \quad \mathrm{i} \text { is odd } \text { and } 1 \leq \mathrm{i} \leq l \\
\frac{\mathrm{i}+2}{2}, \quad \mathrm{i} \text { is even and } 1 \leq \mathrm{i} \leq l
\end{array}\right. \\
& \mathrm{f}\left(\mathrm{u}_{\mathrm{j}}\right)=\left\{\begin{array}{l}
l+\frac{\mathrm{j}+1}{2}, \mathrm{j} \text { is odd and } 2 \leq \mathrm{j} \leq \mathrm{n} \\
l+\frac{\mathrm{n}+\mathrm{j}+1}{2}, \mathrm{j} \text { is even and } 2 \leq \mathrm{j} \leq \mathrm{n}
\end{array}\right.
\end{aligned}
$$

$\mathrm{f}\left(\mathrm{u}_{1}\right)=1$.

For odd $\mathrm{n}$ and even $l$,

$\mathrm{f}\left(\mathrm{v}_{\mathrm{i}}\right)=\left\{\begin{array}{l}\frac{l+\mathrm{i}+3}{2}, \quad \mathrm{i} \text { is odd } \text { and } 1 \leq \mathrm{i} \leq l \\ \frac{\mathrm{i}+2}{2}, \quad \mathrm{i} \text { is even and } 1 \leq \mathrm{i} \leq l\end{array}\right.$

$\mathrm{f}\left(\mathrm{u}_{\mathrm{j}}\right)=\left\{\begin{array}{l}l+\frac{\mathrm{j}+1}{2}, \mathrm{j} \text { is odd and } 2 \leq \mathrm{j} \leq \mathrm{n} \\ l+\frac{\mathrm{n}+\mathrm{j}+1}{2}, \mathrm{j} \text { is even and } 2 \leq \mathrm{j} \leq \mathrm{n}\end{array}\right.$

$\mathrm{f}\left(\mathrm{u}_{1}\right)=1$.

Hence, the $\mathrm{n}+l$ vertices get labels $1,2, \ldots, \mathrm{n}+l$. Therefore, the $(\mathrm{n}, l)-$ dragon is a super edge trimagic total labeling graphs.

Example 2.9. An edge trimagic total labeling of the $(8,6)$ - dragon, $(6,5)-$ dragon, $(5$, $7)$ - dragon and $(7,4)$ - dragon are given in figure 4 , figure 5, figure 6 and figure 7 respectively.

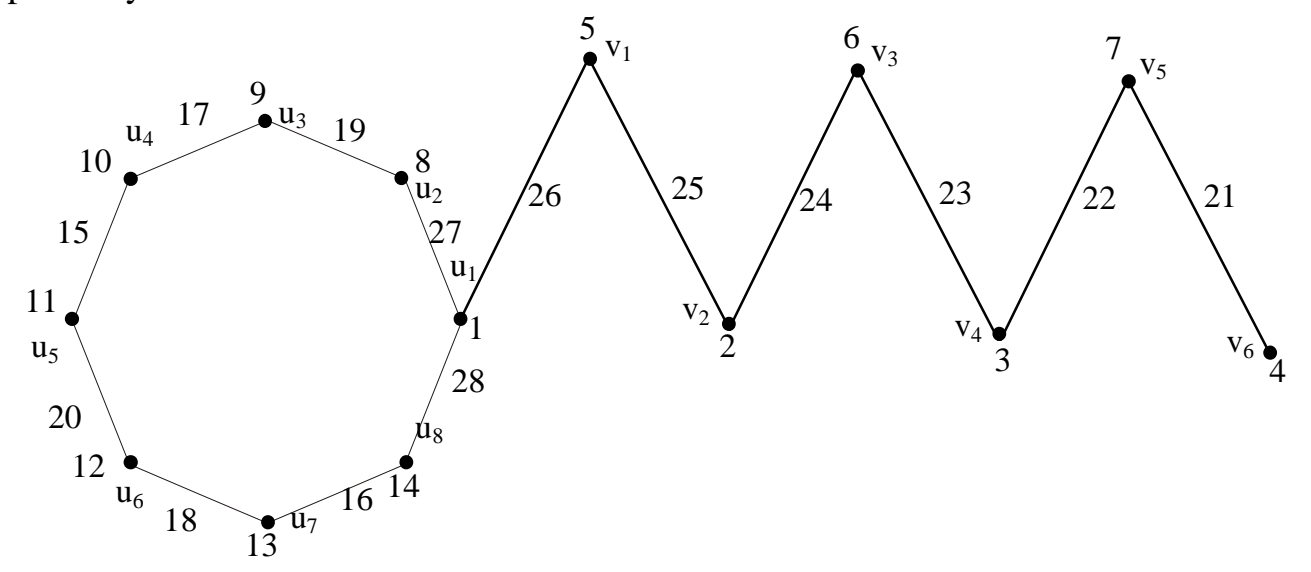

Figure 4: $(8,6)-$ dragon with $\lambda_{1}=32, \lambda_{2}=36$ and $\lambda_{3}=43$ 


\section{Jayasekaran and J. Little Flower}

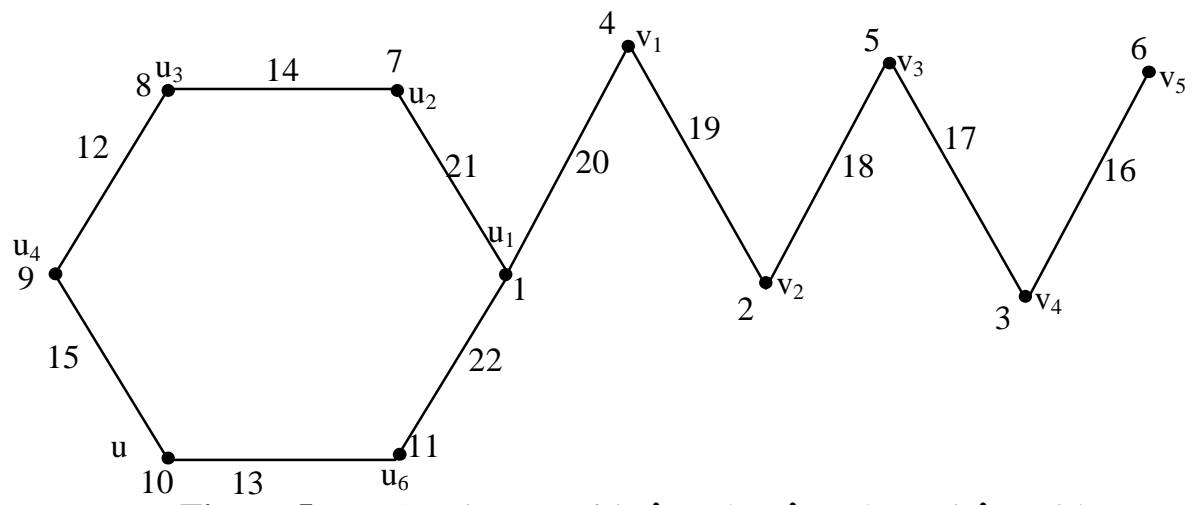

Figure 5: $(6,5)-$ dragon with $\lambda_{1}=25, \lambda_{2}=29$ and $\lambda_{3}=34$

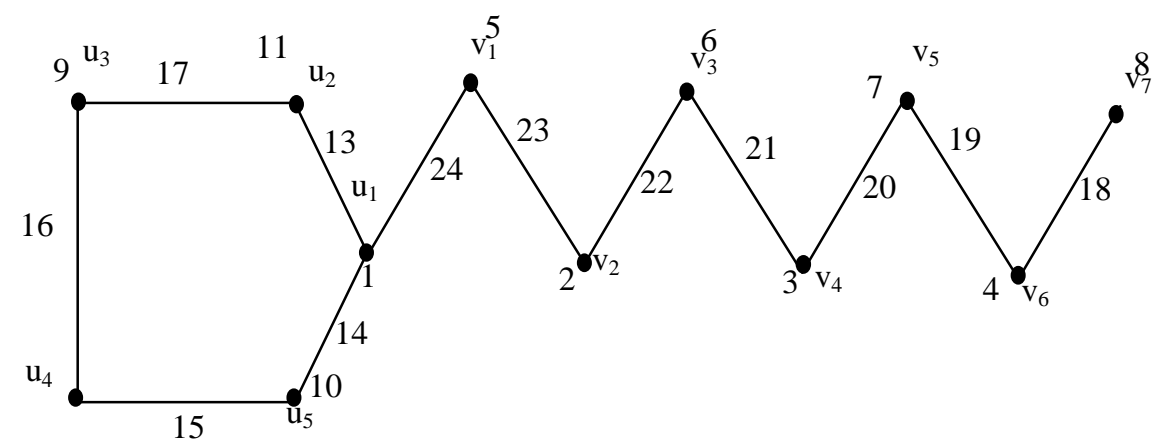

Figure 6: $(5,7)-$ dragon with $\lambda_{1}=30, \lambda_{2}=25$ and $\lambda_{3}=37$

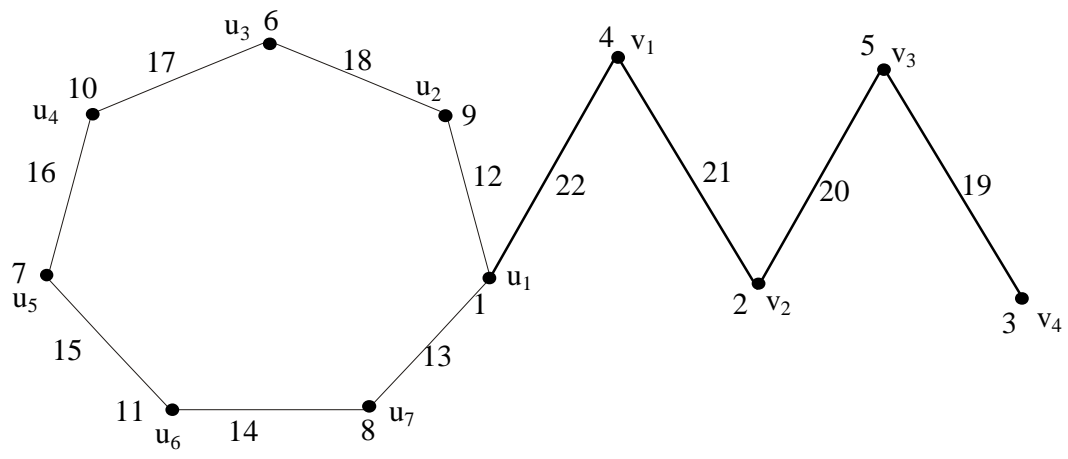

Figure 7: $(7,4)-$ dragon with $\lambda_{1}=27, \lambda_{2}=22$ and $\lambda_{3}=33$

\section{Conclusion}

In this paper, we proved that the Mobius ladder, Book and $(\mathrm{n}, l)-$ dragon are edge trimagic total labeling graphs. Theorem 2.1 shows that Mobius ladder $\mathrm{M}_{\mathrm{n}}$ is an edge trimagic total labeling for odd $n$. One can try to prove that the Mobius ladder $\mathbf{M}_{n}$ graph is an edge trimagic for even $\mathrm{n}$. 
Edge Trimagic Total Labeling of Mobius Ladder, Book and Dragon Graphs

\section{REFERENCES}

1. S. Babith, A. Amarajothi, and J. Basker Babujee, Magic and bimagic labeling for disconnected graphs, International Journal of Mathematics Trends and Technology 3(2) (2012) 86-90.

2. J.B.Babujee, On edge bimagic labeling, Journal of Combinations Information \& System Sciences, 28-29(1-4) (2004) 239-244.

3. L.Girija and A.Elumalai, Edge magic total labeling of the cycle $c_{n}$ with $p_{3}$ chords, Annals of Pure and Applied Mathematics, 8(2) (2014) 175-181.

4. F.Harary, Graph theory, Narosa Publishing house, New Delhi (2001).

5. C.Jayasekaran, M.Regees and C.Davidraj, Edge trimagic labeling of some graphs, Intern. Journal of Combinatorial Graph Theory and Applications, 6(2) (2013) 175186

6. C.Jayasekaran and M.Regees, Edge trimagic in digraphs, Journal of Discrete Mathematical Sciences and Cryptography, 17(4) (2015) 321-335.

7. C.Jayasekaran and J.Little Flower, On edge trimagic labeling of umbrella, dumb bell and circular ladder graphs, Annals of Pure and Applied Mathematics, 13(1) (2017) 73-87.

8. J.A.Gallian, A dynamic survey of graph labeling, The Electronic Journal of Combinatorics, 16 (2013) \#DS6.

9. S.R.Kim and J.Y.Park, On super edge-magic graphs, Ars Combinatoria, 81 (2001) 113-127.

10. A.Kotzing and A.Rosa, Magic valuations of finite graphs, Canad. Math. Bull., 13 (1970) 415-416.

11. K.M.Koh, D.G.Rogers, H.K.Teo and K.Y.Yap, Graphful graphs: Some further results and problems, Congr. Numer., 29 (1980) 559-571.

12. J.Sedlacek, Problem 27. in Theory of Graphs and its Applications, Proc. Symposium Smolenice, June, (1963) 163-167.

13. S.K.Vaidya and N.H.Shah, Some star and bistar related divisor cordial graphs, Annals of Pure and Applied Mathematics, 3(1) (2013) 67-77. 